\title{
Nitric oxide synthase-dependent NADPH-diaphorase activity in the optic lobes of male and female Ceratitis capitata mutants
}

\author{
E. Roda, E. Municchi, E. Conforti, M.B. Pisu, L.M. Gomulski, A.R. Malacrida, G. Bernocchi \\ Dipartimento di Biologia Animale e Istituto di Genetica Molecolare del C.N.R., Sezione di Istochimica e \\ Citometria, Università di Pavia, Italy
}

\begin{abstract}
CC2004, European Journal of Histochemistry
Nitric oxide (NO) is acknowledged as a messenger molecule in the nervous system with a pivotal role in the modulation of the chemosensory information. It has been shown to be present in the optic lobes of several insect species. In the present study, we used males and females from four different strains of the medfly Ceratitis capitata (Diptera, Tephritidae): or; or,wp (both orange eyed); w,M360 and $w$, Heraklion (both white eyed), as models to further clarify the involvement of NO in the mutants' visual system and differences in its activity and localization in the sexes. Comparison of the localization pattern of NO synthase (NOS), through NADPH-diaphorase (NADPHd) staining, in the optic lobes of the four strains, revealed a stronger reaction intensity in the retina and in the neuropile region lamina than in medulla and lobula. Interestingly, the intensity of NADPHd staining differs, at least in some strains, in the optic lobes of the two sexes; all the areas are generally strongly labelled in the males of the or and w,M360 strains, whereas the $w$, Heraklion and or,wp mutants do not show evident sexdependent NADPHd staining. Taken as a whole, our data point to $\mathrm{NO}$ as a likely transmitter candidate in the visual information processes in insects, with a possible correlation among NOS distribution, eye pigmentation and visual function in C. capitata males. Moreover, NO could influence behavioural differences linked to vision in the two sexes.
\end{abstract}

Key words: NOS activity, sex-differences, optic lobes, mutants, insects.

Correspondence: Prof. Graziella Bernocchi, Dipartimento di Biologia Animale, Piazza Botta 10, I-27100 Pavia, Italy. Phone: +39.0382.506327. Fax: +39.0382.506325. E-mail: bern@unipv.it

Paper accepted on November 14, 2003

European Journal of Histochemistry

2004; vol. 48 issue 2 [Apr-Jun]: 141-150
T he gas nitric oxide (NO) is recognized as an important signalling molecule in the nervous system (Moncada et al., 1991; Bredt \& Snyder, 1990), not only in mammals (Bredt \& Snyder, 1990; Garthwaite, 1991; Garthwaite et al., 1988; Snyder, 1992; Vincent \& Kimura, 1992), but also in all the vertebrate groups (Williams et al., 1994; Brüning et al., 1994; Holqvist et al., 1994; Pisu et al., 2002) and in several invertebrates (Martinez, 1995; Müller \& Bicker, 1994; Kurzin et al., 1996; Gibbs \& Truman, 1996; Elphick et al., 1996; Pisu et al., 1999).

$\mathrm{NO}$ is synthesized from L-arginine and molecular oxygen by the enzyme NO-synthase (NOS) (Bredt \& Snyder, 1990), that, using as cofactor NADPH, displays NADPH diaphorase (NADPHd) activity (Garthwaite,1991; Vincent, 1994; Johansson \& Carlberg, 1995); the formation of $\mathrm{NO}$ is $\mathrm{a} \mathrm{Ca}^{2+} /$ calmodulin-dependent process (Garthwaite et al., 1988).

NOS containing neurones are present throughout the mammalian central nervous system (CNS) and recently a gene encoding a protein with $43 \%$ aminoacid identity to rat neural NOS was reported in D. melanogaster (Regulski \& Tully, 1995) providing further evidence that $\mathrm{NO}$ is a signalling molecule in the insect CNS (Elphick et al, 1996). In parallel with mammalian studies, the research in invertebrate focused on role for NO in olfaction (Gelperin et al., 1994; Muller \& Bicker, 1994) and learning and memory (Robertson et al., 1994). All these studies suggest the high conservation of this signalling system throughout the animal kingdom (Martinez, 1995; Eloffson et al., 1993; Ribeiro et al., 1997; Salleo et al., 1996; Elphick et al., 1993a).

$\mathrm{NO}$ is also found at all levels of the vertebrate visual system (Vincent \& Kimura, 1992; Kalamkarov et al., 1993) and it is thought to be implicated in the visual processes and in the visually guided behaviour of some insects (Elphick et al., 1996; 
Bacigalupo et al., 1995; Bicker \& Schmachtenberg, 1997).

Since NO is extremely labile and NOS displays NADPHd activity, frequently the method used to demonstrate nitrinergic elements in the brain consisted in the histochemical reaction for NADPHdiaphorase (NADPHd; Thomas \& Pearse, 1964). The most important and attractive reason for the interest of neuroanatomist and neurobiologist in this technique arose when NADPHd was identified as a marker for neuronal nitric oxide synthase (Hope et al., 1991). Thus, the relative simple NADPHd histochemical technique was widely used to identify NO producing elements in the brain of representatives of all vertebrate classes (Luebke et al., 1992; Brüning, 1993; Panzica et al., 1994; Alonso et al., 1995; Smeets et al., 1997; Arévalo et al., 1995; Muñoz et al., 1996; Alonso et al., 2000). The NADPHd staining method in invertebrate has been validated by purification of the locust NOS and demonstration of co-localization for both NOS and NADPHd activities (Elphick et al., 1994).

In a previous work (Conforti et al., 1999), we have found that NADPHd activity is present in the optic lobes of two different strains of the medfly Ceratitis capitata (Diptera, Tephritidae), a wild type eye colour and a white eye mutant line. Here we give histochemical evidence of the differences in NADPHd activity in the optic lobes of four eye colour mutant strains of $C$. capitata with the aim to further demonstrate a functional relationship between NOS dependent NADPHd activity in the optic lobes and the visual activity of the medfly $C$. capitata. The orange eye (or) and orange eye, white pupa (or,wp) mutants have orange coloured eyes; the white eye $\mathrm{M} 360(w, \mathrm{M} 360)$ strain presents white-yellow eye and the white eye Heraklion $(w$, Heraklion) mutant phenotype lacks eye colour pigmentation.

In particular, we have compared the pattern of NADPHd staining, paying attention to the possible role of a sex-dependent NOS activity in the optic lobes of $C$. capitata.

In fact, it has been previously observed that in many insects some visually guided behaviour patterns differ between the sexes (Franceschini et al., 1981). In particular, in the blowfly Calliphora erythrocephala dimorphism is expressed by differences in the shapes of analogous neurons in males and females, as well as by the presence of some cells in only one sex (Strausfeld, 1980). Sexual dimor- phisms, structural differences between the sexes, have been described in the brains of many vertebrate species.

\section{Materials and Methods}

\section{Insect strains}

Four adult strains of $C$. capitata were used: or; or, wp (both orange eyed); w, M360; w, Heraklion (both white eyed). White eye Heraklion is homozygous for the white eye $\left(w^{2}\right)$ allele of the white locus (w) (Torti et al., 1994). Our experiments were carried out both on males ( 5 specimens per strain) and females ( 5 specimens per strain). The four strains were reared in our laboratory at the same conditions $\left(25^{\circ} \mathrm{C}\right.$ and $65 \% \mathrm{RH}$ under 12 hours light- 12 hours dark conditions).

Visual activities were tested by observation during the routine manteinance and on mating behaviour.

\section{Tissue preparation}

The exoskeleton was dissected from the anterior part of the puparium and from the anterior face of the head to expose the brain and the optic lobes. Heads were fixed with $4 \%$ paraformaldehyde in 0.1 $\mathrm{M}$ phosphate buffer, $\mathrm{pH} 7.35$, for 2 h. After washing in the same buffer for $30 \mathrm{~min}$, they were stored in cryoprotective $25 \%$ sucrose-phosphate buffer overnight at $4^{\circ} \mathrm{C}$ and then frozen in liquid nitrogen. Serial $14 \mu \mathrm{m}$ sections were cut using a cryostat in the frontal plane.

\section{NADPHd histochemistry}

The sections, from different mutants and sexes, were contemporaneously incubated for 1 hour at $37^{\circ} \mathrm{C}$ in the dark in the following medium [modified from Van Noorden and Frederiks, 1992 (Fortini \& Bonini, 2000)]: $0.1 \mathrm{M}$ phosphate buffer, pH 7.35, containing $15 \%(\mathrm{w} / \mathrm{v})$ polyvinyl alcohol, $0.5 \mathrm{mM} \beta$ NADPH (Sigma, M0, USA), 0.2\% Triton-100 and $5 \mathrm{mM}$ nitro blue tetrazolium (NBT, Sigma). After incubation, the sections were rinsed in $0.1 \mathrm{M}$ phosphate buffer, $\mathrm{pH} 7.35$, then mounted on glycerinegelly and viewed/photographed with a Zeiss Axioskop microscope.

Control preparations were stained with the NADPH-diaphorase procedure after: (i) omission of NADPH, (ii) omission of nitro blue tetrazolium, (iii) replacement of NADPH with NAD. In addition, some sections were pre-incubated with $1 \mathrm{mM}$ 
L-NNA ( $\mathrm{N}_{w}$-nitro-L-arginine) dissolved in glicyne$\mathrm{NaOH}$ buffer, $\mathrm{pH}$ 8.5. L-NNA is a specific inhibitor of NOS activity and, consequently, of NO production. Control preparations were not stained, except for $w, M 360$, that showed persistent labelling in the retina.

\section{Evaluation of staining intensity}

In order to make objective evaluations, the staining intensity of each area of the optic lobes was determined by scanning colour photographs of sections with a colour scanner (AGFA ARCUS II) and then using Adobe Photoshop 5.0 for computerized image analysis. The staining intensity was expressed as percentage black in the grey scale. All the intensity values obtained from the control reaction omitting NADPH were subtracted from the correspondent intensity values obtained after specific NADPHd reaction. Data are mean \pm S.D. of five individuals for each stage (five sections for each individual and fifteen measurements for each section). No significant differences in the staining intensity were seen among individuals. The differences between the neuropiles of the phenotypes were analyzed by the Tukey's HSD Multiple Comparisons test.

\section{Results \\ Anatomical description of an insect brain}

The head of an insect is formed from a set of fused segmental units, whose precise number, perhaps seven, has proved difficult to establish. Likewise the head contains a set of segmental ganglia, fused to form a brain, called cerebron. This gangliar complex can be divided into three main areas: proto-, deuto-, and tritocerebro, each one containing nerve centres.

About half of the entire complement of neurones in a Dypteran insect form the optic lobes that serve the compound eyes. In fact, large compound eyes with a facet lens for each pixel in the optical image are a distinctive feature of insects; the basic unit of these compound eye is called ommatidium. Beneath these eyes much of the brain is devoted to vision.

The first stages of vision involve point-by-point transformation and coding of image on the retina. This is done by neurones in the optic lobes, whose columnar architecture of almost crystalline regularity beautifully reflects their function. Signals from the eye pass successively through three gan- glia: the lamina, the medulla and the lobula. In the outer two ganglia - the lamina and the medulla the neurones are arranged to form columnar modules, with a set of cells repeated beneath each ommatidium.

The first and smallest ganglion is the lamina; it receives direct inputs from the retina, as well as feedback from the medulla. Signals passed from the lamina to the medulla, finally enter in the lobula.

\section{Distribution of NOS labelling intensity in the visu- al system of or; or,wp; w,M360; w,Heraklion mutant of C. capitata}

The intensity of NADPHd labelling differs in the optic lobes of the four phenotypes and may be sex dependent.

$w, M 360$ mutant. All the areas of the $w, M 360$ optic lobes were labelled (Figure $1 \mathrm{a}, \mathrm{b}, \mathrm{c}, \mathrm{d}$ ). In particular, a strong intensity of NADPHd staining was found in the retina and in the outer lamina, with respect to the medulla and lobula. However, the immunostaining was stronger in the males. In the male (Figure $1 \mathrm{a}, \mathrm{b}$ ), the labelling is associated with distinct layers of fibers and varicosities along the whole medulla and in the lamina. In the female (Figure $1 \mathrm{c}, d$ ) all the areas of the optic lobe displayed a generally lower staining intensity. In particular, the labelling was higher in the retina and in the lamina whereas the medulla and the lobula were very weakly labelled. Several fibers and varicosities were clearly detectable in the medulla and in the lobula. Both the monopolar cell layer of the outer lamina, and the cell bodies adjacent to the medulla were weakly stained in both sexes.

or mutant. The optic lobes of males and females of the or strain displayed different NADPHd staining intensities (Figure $2 \mathrm{~b}, \mathrm{~d}$ ). All the areas of the optic lobes were strongly labelled in the or male (Figure $2 \mathrm{~b}$ ). The staining was most intense in the retina and in the lamina, whereas the inner neuropiles displayed less NADPHd staining. The photoreceptors showed intense immunostaining and in the lamina and in the outer medulla fibres and varicosities were also clearly labelled. The monopolar cell layer of the outer lamina was weakly stained. On the contrary, in the or females (Figure $2 \mathrm{~d}$ ) only the retina displayed intense labelling; the staining was weak in the lamina and it decreased in the medulla and in the lobula. Low immunostaining was present in the monopolar layer and only sporadically a few fibers and varicosities were labelled in 

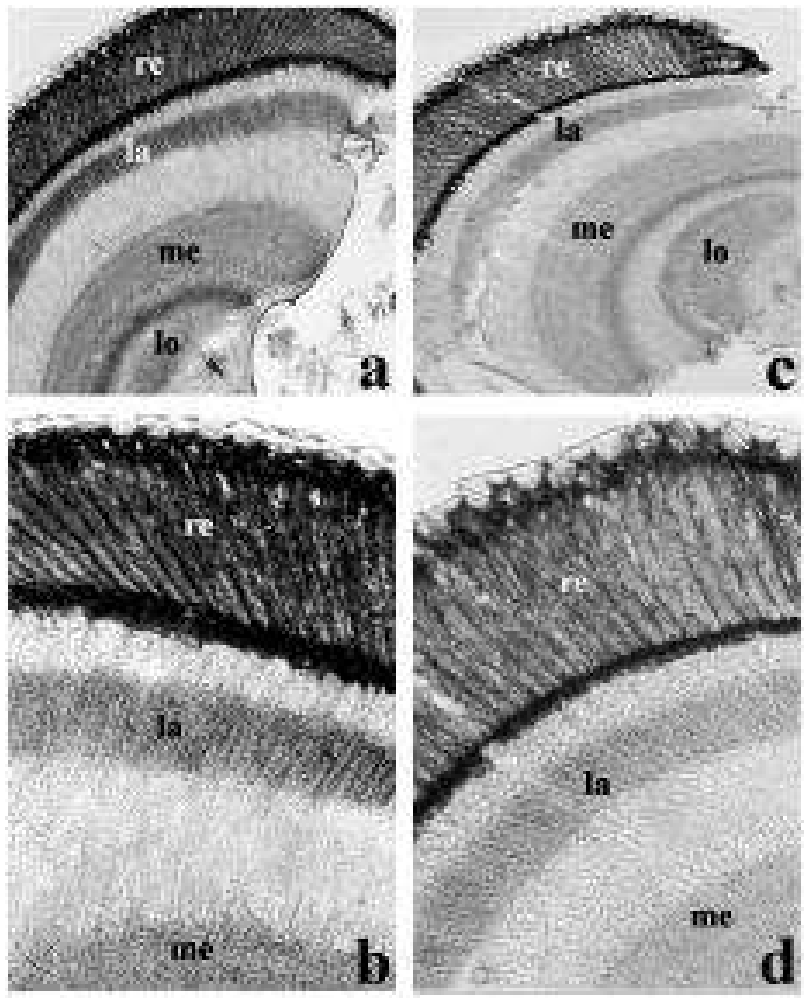

Figure 1. Distribution of NADPHd staining in the optic lobes of the w,M360 mutant of $C$. capitata. (a, b) Frontal sections, adult male. A generally strong NADPHd staining is evident throughout the lobes. The staining is very intense in the retina (re); labelling is also present in the three neuropilar areas: lamina (la), medulla (me) and lobula (lo), where it is locally associated with distinct cell layers. In the medulla fibres and varicosites are also clearly labelled. (c, d) Frontal sections, adult female. All the areas of the lobes show NADPHd staining. A lower labelling intensity, with respect to the male, is evident both in retina and in all neuropilar regions. $(a, c)$ : objective 10x1.6; (b, d): objective 40x.

the outer medulla.

$w$, Heraklion mutant. The $w$, Heraklion optic lobes were stained by NADPHd reaction (Figure $2 \mathrm{a}, \mathrm{c}$ ) and, like the or and w, M360 mutants, they displayed different labelling intensities in males (Figure 2 a) and females (Figure $2 \mathrm{c}$ ). In particular, lower intensity of staining was found in the medulla and in the lobula, with respect to the retina and the lamina in both sexes. However, the retina and the lamina were strongly labelled in the male. Cell bodies of the monopolar layer were not stained, while in the lamina and outer medulla several fibers and varicosities were labelled.

or,wp mutant. The or,wp optic lobes did not display different staining intensities in males and females (not shown in figures). The labelling was

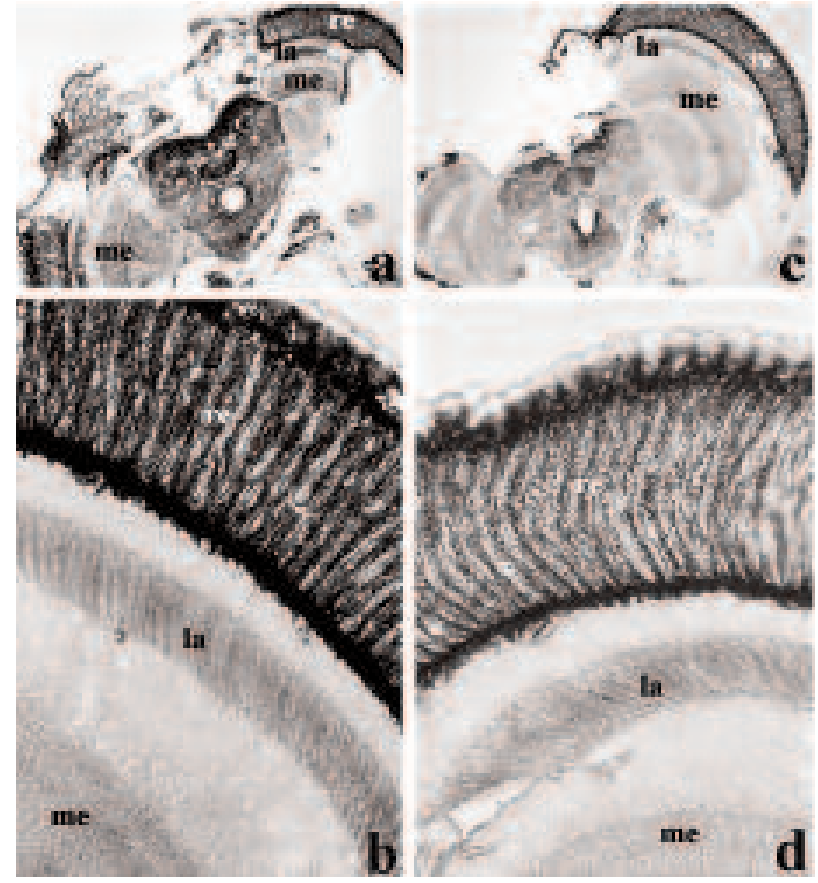

Figure 2 Distribution of NADPHd staining in the optic lobes of w,Heraklion mutant of C. capitata. (a, b) Frontal section, adult male. NADPHd staining is evident throughout the lobes. The staining is very strong in the retina (re); labelling is also present in the neuropilar regions lamina (la) and medulla (me). (c, d) Frontal section, adult female. All the areas of the lobes show NADPHd staining. A slightly lower labelling intensity, in comparison with the male, is evident both in retina and in the lamina. (a, c): objective 10x; (b, d):objective 40x.

generally strong, particularly in the retina and in the lamina.The lamina cartridge showed strong labelling in both sexes. In the outer medulla, fibers and varicosities were stained while the monopolar cell layer of the outer lamina was weakly labelled.

\section{Intensity values of NADPHd activity of or; or,wp; w,M360 and w,Heraklion mutant optic lobes}

The variation of NADPHd staining intensity in the optic lobes of males and females of the four strains was considered (Figure 3). The pattern of staining distribution was similar in the optic lobes of the four phenotypes: the retina and lamina generally displayed a higher degree of staining than the medulla and lobula.

The comparison between males and females of 


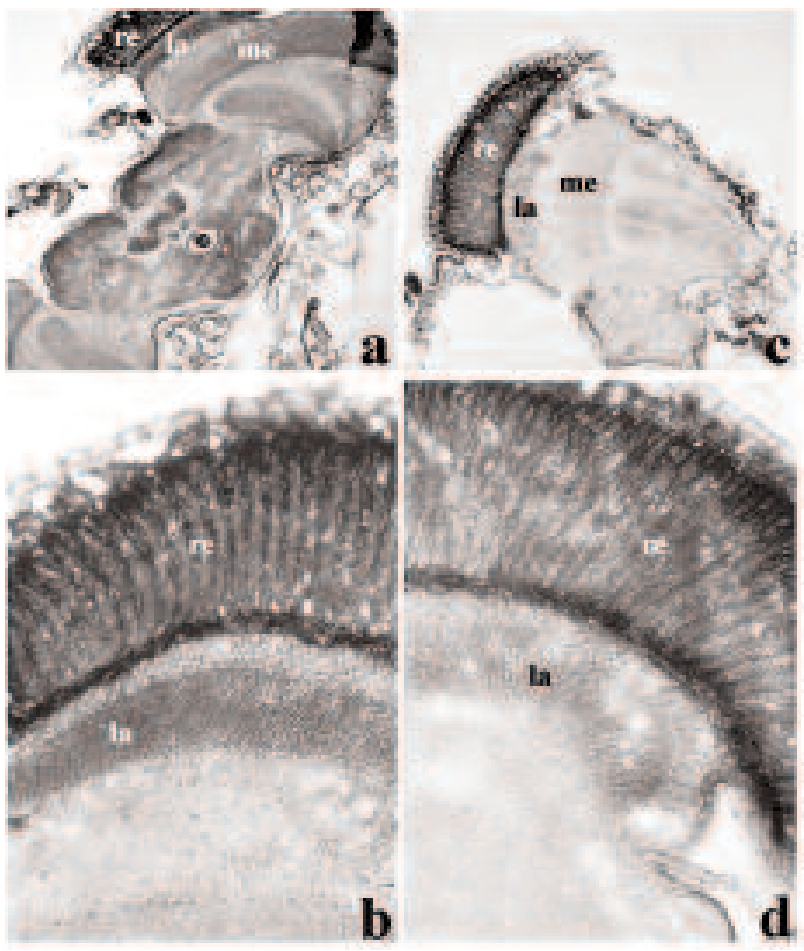

Figure 3. Distribution of NADPHd staining in the optic lobes of or mutant of C. capitata. (a, b) Frontal section, adult male. NADPHd staining is observed throughout the lobes. A strong labelling is found both in the retina (re) and in lamina (la). (c, d) Frontal section, adult females. The staining is moderate in all the areas. A decreased intensity of labelling, with respect to the male, is observed both in retina (re) and in lamina (la). (a, c): objective 10x1.2; (b, d): objective 40x. each strain and among all the flies of the same sex (Figure 4) showed that all the areas of the optic lobes were generally strongly labelled in the male of $w_{1}$ M 360 strain and, much more, in the or mutants, whereas significant differences in intensities were not evident in the or,wp strain. In the $w$, Heraklion strain, the female showed a slightly lower staining with respect to the male, but this difference was not highly significant. Moreover, it appeared that, while in the male the intensity of NADPHd staining increased when the eye pigmentation intensified, in the female this relationship was not present.

\section{Discussion}

\section{Technical considerations}

Since the enzymatic activity of NOS requires a cofactor NADPH, histochemistry has been used extensively to localize NOS through the reduction of tetrazolium salts to an insoluble formazan reaction product. The large use of NADPHd staining procedure indicates that it is sensitive and specific for several tissues, after careful fixation with formaldehyde (Eldred, 2000) and therefore it is more reliable in the detection of NOS in the animal kingdom. In fact we have recently demonstrated NOS immunoreactivity in Ceratitis $w$ e w+ strains (Conforti et al., 2002); however, results required
MALES

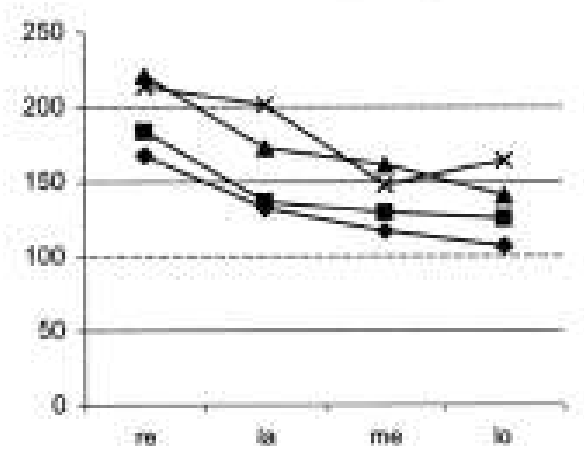

FEVALES

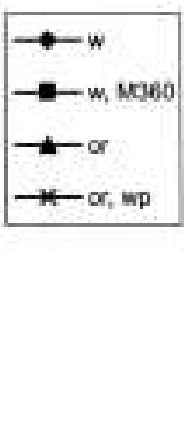

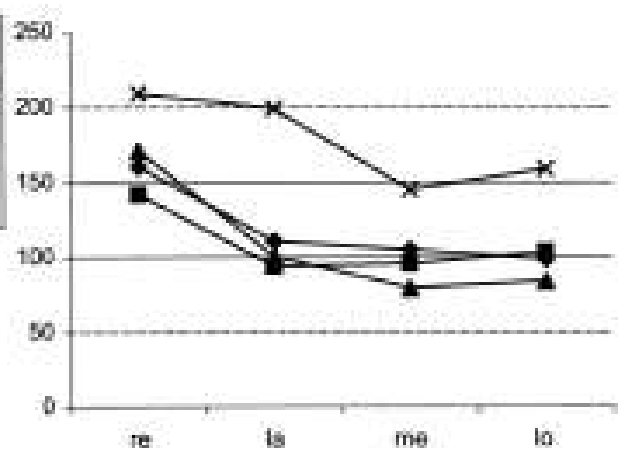

Figure 4. Staining intensity values (mean \pm S.D.) of NADPHd activity of or, or, wp, w,M360 and w,Heraklion mutants optic lobes.The pattern of staining distribution was similar in the optic lobes of the four phenotypes: retina and lamina generally displayed a higher degree of staining than the other neuropilar regions. The comparison between males and females of each strain showed that all the areas of optic lobes were strongly labelled in the males of $w, M 360$ and much more, in the males of or mutant. In or,wp mutants, the difference between the two sexes was not significant. In $w, M 360$ optic lobes, the variations of staining intensity between males and females were significant ( $p<0.01$ ) in retina, lamina and medulla, whereas lobula showed a low significance $(p<0.05)$. In or optic lobes, all variations of staining intensity between males and females were highly significant $(p<0.001)$. Therefore, in $w$,Heraklion optic lobes, the variations of staining intensity were not significant, except for retina and lamina that showed a low significance ( $<<0.05$ ). 

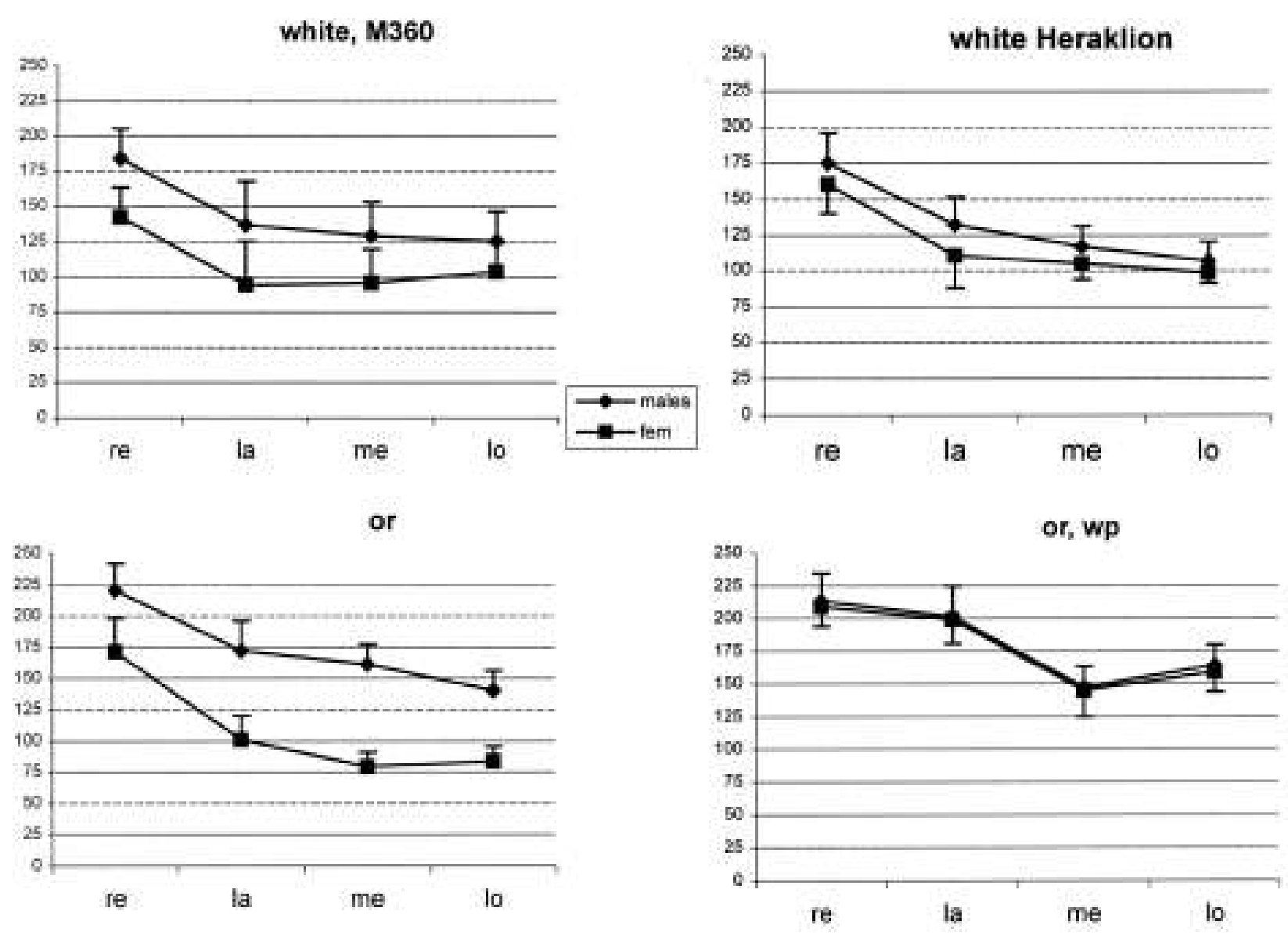

Figure 5. Staining intensity values (mean \pm S.D.) of NADPHd activity of or, or, $w p, w$,M360 and $w$,Heraklion mutants optic lobes. Comparison between all the flies of the same sex: while in the males the intensity of NADPHd staining increased when the eye pigmentation intensified, in the females this relationship was not present.

hard criticism for their evaluations, that was possible since we have already found clear differences of NADPHd reactivity between $w$ and $w+$ strains (Conforti et al., 2001).

\section{NOS-dependent NADPHd pattern and role in the male optic lobes}

NOS has been characterized in brain of various insects, such as the honeybee Apis mellifera, the fruitfly Drosophila melanogaster, the locust Schistocerca gregaria and the cricket Acheta domestica (Elphick et al., 1996; Müller, 1994) but the centres of visual information processing exhibit very different levels of staining among the species. While in Drosophila NADPH diaphorase staining appears to be almost absent in visual neuropiles, Apis exhibits an intermediate and Acheta and Schistocerca a very strong labelling in the visual neuropiles (Müller, 1997). The extreme differences in NADPH diaphorase labelling in the visual system of the various insects suggest a role of the NO system which is more likely characteristic for certain species than a conserved function. Here we have used the NADPHd histochemical technique on a single species, the medfly $C$. capitata, comparing the staining pattern of the optic lobes among four eye colour mutant strains, i.e., or; or, wp, w, M360 and $w$, Heraklion. Previously we demonstrated the piv- 
otal role of nitric oxide (NO) in the modulation of the chemosensory information, that seems to be implicated also in visual processes and visually guided behaviour of many insects, using NADPHd staining on two different strains of the medfly $C$. capitata (Conforti et al., 1999).

Our study revealed a peculiar pattern of NADPHd staining in the optic lobes of the species with respect to those previously observed in other insects. These results showed that the staining pattern was similar in the optic lobes of the four strains: the retina and lamina generally displayed a higher degree of staining than the medulla and lobula.

The most remarkable feature was the difference in the labelling intensity of the visual tissue among the four eye colour phenotypes. The optic lobes of the four strains displayed high staining along the whole retina and lamina, whereas the medulla and the lobula showed lower NADPHd labelling. However, the optic lobes of the phenotypes with less pigmented eye ( $w$, Heraklion \& $w, \mathrm{M} 360)$ displayed a weaker NADPHd staining, at least in the male (see below), than those of the phenotypes with a more intense eye pigmentation.

Independent studies of artificial populations of $D$. melanogaster agree that where wild type and laboratory eye colour mutants are in competition, the mutants are at a distinct disadvantage (Torti et al., 1997). There are observations which point to mating behaviour as a source of disadvantage to the competing mutant flies: it was shown that wild type males are more effective than mutant males when competing for the same females (Geer \& Green, 1962). Also in response to light $D$. melanogaster eye colour mutants vary in their ability to orient themselves, compared to wild type eye colour flies (Fingerman, 1952; Spieth \& Hsu, 1950). These results emphasized the conclusion that a difference in visual recognition or discrimination accounts for the advantage of one type of male in the light. Moreover, visual activities were tested during the routine maintenance and on mating behaviour of wild type $(w+)$ and white eye Heraklion ( $w$, Heraklion) mutant of $C$. capitata. These tests showed the higher competitiveness of the wild type males in their mating choice, compared to the white eye mutant males (Conforti et al., 1998; Conforti et al., 1999). Based on these observations, the marked NADPHd staining difference among or, or,

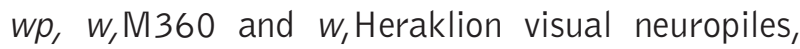
which are specifically devoted to the analysis and elaboration of the visual signals, suggested a role for NO in the regulation of the visual information processes of $C$. capitata. Moreover, the observation of the correlation between the NADPHd staining and eye pigmentation suggested a possible relationship between the intensity of eye pigmentation and the protection of the visual system.

In particular, a neurodegenerative disease, analogous to the human condition retinitis pigmentosa, was analyzed in Drosophila (Fortini \& Bonini, 2000). Mutant variants of several Drosophila photoreceptor cell-specific proteins, including rhodopsin, structural proteins and other factors needed for rhabdomere integrity all cause gradual, light-independent degeneration. Therefore the intensity of eye pigmentation could favour the protection of the visual system.

\section{Relationship between NOS-dependent NADPHd and sex}

The intensity of NADPHd staining differed in the optic lobes of the four phenotypes and moreover it appeared to differ, at least in some strains, between the two sexes. All the areas of the optic lobes were generally strongly labelled in the male, except for the or, wp mutant, which did not display significant labelling differences between sexes. Considering the pattern of labelling intensity distribution in the four strains, we observed that in the male the intensity of NADPHd staining increased when the eye pigmentation intensified. As previously stated, these results suggested a possible relationship among NOS, eye pigmentation and visual information processes in the $C$. capitata male. Otherwise, the female displayed a very heterogeneous NADPHd labelling and no correlation between eye pigmentation and NOS was shown. In comparison with the males, the females of the or; $w, M 360$ and $w$, Heraklion strains displayed weak staining, whereas the or,wp females appeared strongly labelled. In particular, the weak staining of the optic lobes of the or mutant, characterized by orange eye coloration, did not allow a correlation between intensity of eye pigmentation and NOS in the female. These results could suggest that nitric oxide production may be dependent upon behavioural differences linked to vision function in the two sexes of $C$. capitata.

Several works (Franceschini et al., 1981; Strausfeld, 1980) analyzed sexual dimorphism in insect vision: some visually guided behaviour pat- 
terns differ between the sexes and presumably information processing by nerve cells also differs in males and females. Recent anatomical studies of houseflies Musca domestica have demonstrated that certain visual neurons are present only in males. Also in the blowfly $C$. erythrocephala another kind of sexual difference in the neural architecture of the visual system was reported. In this fly dimorphism was expressed by differences in the shapes of analogous neurons in males and females, as well as by the presence of some cells in only one sex (Franceschini et al., 1981).

The widely accepted view that $\mathrm{NO}$ also plays an essential role also in mating behaviour and that there is an evident relationship between NOS and sexual dimorphism, were confirmed by Hadeishi \& Wood's work (Hadeishi \& Woods, 1996) that reported that $\mathrm{NO}$ is present in the mating behaviour circuitry of the brain of the male Syrian hamster. Obviously chemosensory and hormonal stimuli are essential for mating in the male Syrian hamster; $\mathrm{NO}$ is implicated in the regulation of male sexual behaviour, and NOS is present in the limbic system. Moreover, NO has a pivotal role in male courtship behaviour of the urodele crested newt (Triturus carnifex) but not that of the females (Zerani \& Gobbetti,1996).

A number of experiments also displayed sexual differences in the morphology and the function of neurons in the brains of vertebrates and in particular mammals (Goto \& Goto, 2000; Grachev \& Apkarian, 2000; Cooke et al., 1998; Raisman \& Field, 1973; Nottebohm \& Arnold, 1976).

In the human brain sexual dimorphism was linked not only to anatomical but also to chemical features. The orbital frontal cortex and sensorimotor cortex showed gender dependence, with women demonstrating increased metabolite concentrations compared to man (Nopoulos et al., 2000). Data indicate that the morphology and chemistry of brain may be sex-dependent.

\section{Acknowledgments}

This research was supported by F.A.R. grants to G. Bernocchi.

\section{References}

Alonso JR, Arévalo R, Porteros A, Briñòn JG, Garçia-0jeda E, Aijòn $J$. NADPH-diaphorase staining in the central nervous system. Neurosci. Protocols 1995;95:1-11.

Alonso JR, Arévalo R, Weruaga $E$, Porteros JG, Briñòn JG, Aijòn J. Comparative and developmental neuroanatomical aspects of the
NO system. In: Steinbush HWM, De Vente J, Vincent SR (Eds.), Functional Neuroanatomy of the Nitric Oxide System. Elsevier, Amsterdam, 2000. p. 51-109.

Arévalo R, Alonso JR, Garçia OE, Briñòn JG, Crespo C, Aijon J. NADPH-diaphorase in the central nervous system of the tench (Tinca tinca L. 1758). J Comp Neurol 1995;352:398-420.

Bacigalupo J, Bautista DM, Brink DL, Hetzer JF, O'Day PM. CyclicGMP enhances light induced excitation and induces membrane currents in Drosophila retinal photoreceptors. J Neurosci 1995; 15:7196-200.

Bicker G, Schmachtenberg 0. Cytochemical evidence for nitric oxide cyclic GMP signal transmission in the visual system of the locust. Eur J Neurosci 1997:9:189-93.

Bredt DS, Snyder SH. Isolation of nitric oxide synthase, a calmodulin requiring enzyme. Proc Natl Acad Sci USA 1990;87:682-5.

Brüning G. Localization of NADPH-diaphorase in the brain of the chicken. J. Comp. Neurol. 1993;334:192-208.

Brüning $G$, Wiese $S$, Mayer B. Nitric oxide synthase in the central nervous system of the turtle Pseudemys scripta elegans. J Comp Neurol 1994;348:183-206.

Conforti E, Malacrida AR, Gasperi G, Bernocchi G. II sistema visivo di Ceratitis capitata durante lo sviluppo: evidenza citochimica dell'ossido d'azoto. Proc. 59 Nat. Congr. U.Z.I., 1998, p.97.

Conforti E, Torti C, Malacrida AR, Bernocchi G. Mature and developing visual system of Ceratitis capitata (Diptera, Tephritidae): histochemical evidence of nitric oxide synthase in the wild type and the white eye mutant strains. Brain Res 1999;843:1-11.

Conforti $E$, Barni $S$, Pisu MB, Vaccarone $R$, Malacrida AR, Bernocchi $G$. Cell death may regulate visual functionality in the retina of adults of the dipteran Ceratitis capitata. Neurosci Lett 2001;317:156-60.

Conforti E, Pisu MB, Guioli S, Malacrida AR, Bernocchi G. Nitric oxide synthase-like and calmoduline-likein the visual system of wild type and white eye mutant strains of Ceratitis capitata (Diptera, Tephritidae). Ital J Zool 2002;69:285-94.

Cooke B, Hegstrom CD, Villeneuve LS, Breedlove SM. Sexual dimorphism of the vertebrate brain: Principles and Mechanism. Front Neuroendocrinol 1998;19:323-62.

Eldred WD. Nitric oxide in the retina. In: Steinbusch HWD, De Vente J, Vincent SR (Eds.), Functional Neuroanatomy of the Nitric Oxide System. Elsevier, Amsterdam; 2000. p.111-45.

Eloffson R, Carlberg M, Moroz L, Nezlin L, Sakharov D. Is nitric oxide (NO) produced by invertebrate neurons? NeuroReport 1993; 4:279-82.

Elphick MR, Green IC, O'Shea M. Nitric oxide synthesis and action in an invertebrate brain. Brain Res 1993;619:344-6.

Elphick MR, Green IC, O'Shea M. Nitric oxide signalling in invertebrate nervous system. In: Borkovec $A B$, and Loeb MJ (eds.), Insect Neurochemistry and Neurophysiology. CRC Press., Boca Raton; 1993. p. 129-32.

Elphick MR, Green IC, $\mathrm{O}^{\prime}$ Shea M. Nitric oxide signilling in the insect nervous system. In: Borkovec $A B$, and Loeb MJ (eds.), Insect Neurochemistry and Neurophisiology. CRC Press, Boca Raton; 1994. p. 129-32.

Elphick MR, Williams L, O'Shea M. New features of the locust optic lobe: evidence of a role for nitric oxide in insect vision. J Exp BioI 1996;199:2395-407.

Fingerman M. The role of the eye pigments of Drosophila melanogaster in photic orientation. J Exp Zool 1952;120:131-64.

Fortini ME, Bonini NM. Modeling human neurodegenerative diseases in Drosophila.TIG 2000;16:161-7.

Franceschini N, Hardie R, Ribi W, Kirschfeld K. Sexual dimorphism in a photoreceptor. Nature 1981;291:241-4.

Garthwaite J. Glutamate, nitric oxide and cell-cell signalling in the nervous system. Trends Neurosci 1991;14:60-7.

Garthwaite J, Charles SL, Williams RC. Endothelium-derived relaxing factor release on activation of NMDA receptors suggests a role as intercellular messenger in the brain. Nature 1988; 336:385-7.

Geer BW, Green MM. Genotype, phenotype and mating behaviour of Drosophila melanogaster. Am Nat 1962;96:175-81.

Gelperin A. Nitric oxide mediates network oscillations of olfactory interneurons in a terrestrial mollusc. Nature 1994;369:61-3. 
Gibbs SM, Truman JW. Nitric oxide and cyclic GMP regulate retinal patterning in the optic lobe of Drosophila. Neuron 1998;20:8393.

Goto J, Goto N. Sexual dimorphism of the human cerebral pallium. Okajimas Folia Anat Jpn 2000;77:35-7.

Grachev ID, Apkarian AV. Chemical heterogeneity of the living human brain: a proton MR spectroscopy study on the effects of sex, age, and brain region. Neuroimage 2000;11:554-63.

Hadeishi Y, Woods RI. Nitric oxide synthase in mating behaviour of male Syrian hamster brain. J. Neurobiol 1996;30:480-92.

Holmqvist BI, Ostholm T, Alm P, Ekstrom P. Nitric oxide synthase in the brain of a teleost. Neurosci Lett 1994;171:205-8.

Hope BT, Michael JF, Knigge KM, Vincent SR. Neuronal NADPHdiaphorase is a nitric oxide synthase. Proc Natl Acad Sci USA 1991;88:2811-4.

Johansson KUI, Carlberg M. NO-synthase: what can research on invertebrates add to what is already known? Adv Neuroimmunol 1995;5:431-42.

Kalamkarov GR, Shevchenko TF, Balaev AG, Vanin AF. N0-synthase in the vertebrate retina. Endothelium 1 1993; Suppl:s57.

Kurzin B, Roberts I, Peunova N, Enikolopov G. Nitric oxide regulates cell proliferation during Drosophila development. Cell 1996; 87:639-49.

Luebke JI, Weider JM, McCarley RW, Green RW. Distribution of NADPH-diaphorase positive somata in the brain-stem of the monitor lizard Varanus ezanthematicus. Neurosci Lett 1992; 148:129-32.

Martinez A. Nitric oxide synthase in invertebrates. Histochem J 1995;27:770-6.

Moncada S, Palmer RMJ, Higgs EA. Nitric oxide: physiology, pathophysiology and pharmacology. Pharmacol Rev 1991;43:109-42.

Müller U, Bicker G. Calcium-activated release of nitric oxide and cellular distribution of nitric oxide-synthesizing neurons in the nervous system of the locust. J Neurosci 1994;14:7521-8.

Müller U. $\mathrm{Ca}_{2}^{+} /$calmodulin-dependent nitric oxide synthase in Apis mellifera and Drosophila melanogaster. Eur J Neurosci 1994; 6: 1362-70.

Müller U. The nitric oxide system in insects. Prog Neurobiol 1997; 51:363-81.

Muñoz M, Muñoz A, Marin 0, Alonso JR, Arévalo R, Porteros A, Gonzales A. Topographical distribution of NADPH-diaphorase activity in the central nervous system of the frog, Rana perezi. J. Comp. Neurol 1996;367:54-69.

Nopoulos P, Flaum M, O'Leary 0, Andreasen N. Sexual dimorphism in the human brain: evaluation of tissue volume, tissue composition and surface anatomy using magnetic resonance imaging. Psychiatry Res 2000;98:1-13.

Nottebohm F, Arnold A. Sexual dimorphism in vocal control areas of the songbird brain. Science 1976;194:211-3.

Panzica GC, Arévalo R, Sànchez F, Alonso JR, Aste N, Viglietti PC, et al. Topographical distribution of reduced nicotinamide adenine dinucleotide phosphate-diaphorase in the brain of the Japanese quail. J Comp Neurol 1994;342:97-114.
Pisu MB, Conforti E, Fenoglio C, Necchi D, Scherini E, Bemocchi G. Nitric oxide-containing neurons in the nervous ganglia of Helix aspersa during rest and activity: immunocytochemical and enzyme histochemical detection. J Comp Neurol 1999;409:27484.

Pisu MB, Conforti E, Botta L, Valli P, Bernocchi G. Nitric oxide synthase in the frog cerebellum: response of Purkinje neurons to unilateral eighth nerve transection. Anat Rec 2002;268:73-83.

Raisman G, Field PM. Sexual dimorphism in the neuropile of preoptic area of the rat and its dependence on neonatal androgen. Brain Res 1973;54:1-29.

Regulski M, Tully T. Molecular and biochemical characterization of dNOS: A Drosophila $\mathrm{Ca}^{+} / \mathrm{calmodulin}$-dependent nitric oxide synthase. Proc Natl Acad Sci USA 1995;92:9072-6.

Ribeiro JMC, Hazzard JMH, Nussenzveig RH, Champagne DE, Walker FA. Reversible binding of nitric oxide by a salivary heme protein from a bloodsucking insect. Science 1993;260:539-541.

Robertson JD, Bonaventura J, Kohm AP. Nitric oxide is required for tactil learning in Octopus vulgaris. Proc R Soc Lond B 1994;256: 269-73.

Salleo A, Musci G, Barra PFA, Calabrese L. The discharge mechanism of acontial nematocytes involves the release of nitric oxide. J Exp Biol 1996;199:1261-7.

Smeets W, Alonso JR, Gonzàles A. Distribution of NADPHdiaphorase and nitric oxide synthase in relation to catecholaminergic neuronal structures in the brain of the lizard Gekko gecko. J Comp Neurol 1997;377:121-41.

Snyder SH. Nitric oxide: first in a new class of neurotransmitter? Science 1992;257:494-6.

Spieth T, Hsu TC. The influence of light of seven species of the Drosophila melanogaster species group. Evolution 1950;4:31625.

Strausfeld NJ. Male and female visual neurones in dipterous insects. Nature 1980;283:381-3.

Thomas E, Pearse AGE. The solitary active cells: histochemical demonstration of damage-resistant nerve cells with a TPN-diaphorase reaction. Acta Neuropathol 1964;3:238-49.

Torti C, Malacrida AR, Yannopoulos G, Louis C, Gasperi G. Hybrid disgenesis-like phenomena in the medfly Ceratitis capitata (Diptera, Tephritidae). J Hered 1994;85:92-9.

Torti C, Gomulski LM, Malacrida AR, Capy P, Gasperi G. Genetic and molecular investigation in the endogenous mobile element of non-drosophilid fruitflies. Genetica 1997;100:119-29.

Vincent SR, Kimura H. Histochemical mapping of nitric oxide synthase in the rat brain. Neuroscience 1992;46:755-84.

Vincent SR. Nitric oxide: a radical neurotransmitter in the central nervous system. Prog Neurobiol 1994;42:129-60.

Williams CV, Nordquist D, McLoon SC. Correlation of nitric oxide synthase expression with changing patterns of axonal projections in the developing visual system. J Neurosci 1994;14:1746-55.

Zerani M, Gobbetti A. NO sexual behaviour in newts. Nature 1996; 382:31. 
E. Roda et al. 